\title{
EITA! Uma Proposta Inovadora para um Programa de Pós-Graduação Latu-Sensu em Educação, Inovação e Tecnologia Aplicada
}

\author{
César França, Taciana Pontual Falcão, Marcelo Luiz Monteiro Marinho, \\ Marcos Cardoso, Obionor Nóbrega, Ricardo Souza, Suzana Sampaio \\ Departamento de Informática e Estatística (DEINFO) \\ Universidade Federal Rural de Pernambuco. \\ coordenação.eita@ufrpe.br
}

\begin{abstract}
Nowadays, the demand for innovation of educational processes is consensual. However, there are only a few initiatives in Brazil that consider innovative practices as part of the teacher training program. In this article, we report our experience developing a curriculum proposal for a Latu Sensu graduate course in Education, Innovation and Applied Technology (EITA). We hope, by sharing this proposal, to convey the message that such innovation must be led by the teachers, who for this reason must develop appropriate skills in order to create and undertake their own innovations, acting thus as transformational agents of the educational reality of the country.
\end{abstract}

Resumo. A demanda contemporânea pela renovação dos processos educacionais é consensual. No entanto, ainda são poucas as iniciativas no Brasil a considerarem práticas de inovação na formação de professores. Neste artigo, relatamos a nossa experiência de elaboração de uma proposta curricular para um curso de pós-graduação Latu Sensu em Educação, Inovação e Tecnologia Aplicada (EITA). Esperamos, ao compartilhar esta proposta, transmitir a mensagem de que esta renovação deve ser liderada pelos próprios professores, que para isto demandam o desenvolvimento de competências adequadas para criar e empreender suas próprias inovações, $e$ assim atuar como agentes transformadores da realidade educacional no país.

\section{Introdução}

Desde o início do século 21, a tecnologia adquiriu uma alta relevância social, transformando o cotidiano das pessoas, e colocando a inclusão digital como fator primordial de mudanças socioculturais e governamentais, com a criação de regras para disponibilização de conteúdo, acesso à informação e direitos dos usuários. Ao largo desta evolução, processos de ensino-aprendizagem também avançaram de forma significativa. Diversas intervenções tecnológicas educacionais foram propostas na literatura, desde métodos mais clássicos (ex. aulas expositivas com projeções interativas), até estratégias mais criativas (ex. aprendizagem adaptativa). Porém, atualmente, os tipos mais clássicos são usados em 90\% dos casos [Mazzioni 2013]. É comum encontrar justificativas que se fundamentam no baixo acesso e pouco domínio de tecnologias nos processos de sala de aula. Porém, um recente levantamento do Comitê Gestor da Internet no Brasil, encomendado pela UNESCO, aponta que: 
“... embora a adoção das TIC tenha produzido mudanças visíveis na rotina escolar, o uso de tais recursos ainda não produziu transformações substantivas nos processos de ensino $e$ aprendizagem" [NIC.br 2016, p. 100]

Sendo assim, o desafio atual envolve também fazer com que educadores estejam abertos à mudança, repensem as suas práticas, e enfrentem o paradigma das instituições de ensino como fontes de transmissão unilateral de informação, possibilitando o uso de novas propostas interdisciplinares, metodologias interativas e colaborativas, e permitindo-se a exploração de novos espaços que vão além dos limites da sala de aula [Kenski 2015]. Ou seja, os educadores precisam desenvolver mais sistematicamente atitudes inovadoras [Leal et al. 2011]. A importância de desenvolver a atitude inovadora vem sendo cada vez mais reconhecida no Brasil, que em 2015, cresceu em 3 pontos percentuais no grau de empreendedorismo geral da população [Kelley et al. 2016].

Partindo deste pressuposto, o presente artigo relata uma proposta para um curso de pós-graduação Latu Sensu em Educação, Inovação e Tecnologia Aplicada (EITA). Como fundamentação, conduzimos uma análise do panorama de programas de pósgraduação em Educação e Tecnologia no Brasil e no exterior, Em seguida desenvolveuse uma proposta curricular que rompe o paradigma de que a tecnologia por si só é suficiente para melhorar as práticas educacionais, e adota um processo que se distancia de abordagens tradicionalistas, alinhado com a Aprendizagem Baseada em Competências [Mendonça 2007], ao combinar uma prática instrucional atual (ProblemBased Learning [Savery e Duffy 2001]) com um processo de inovação (Design Thinking [Brown 2008]) sob uma abordagem pedagógica construtivista (Ciclo da Aprendizagem Vivencial [Kolb 1984]).

O artigo está estruturado da seguinte maneira: a Seção 2 apresenta o referencial teórico; a Seção 3 relata análises de programas de educação e tecnologia; a Seção 4 detalha a proposta curricular para o EITA; e por fim, a Seção 5 apresenta conclusões e direcionamentos futuros.

\section{Referencial Teórico}

\subsection{Inovação e suas Dimensões de Impacto na Educação}

Inovação refere-se à “implementação de produtos, processos, mercados, métodos, práticas de negócios, organizações ou relações externas novos ou significantemente melhorados" [OECD 2005, p. 46]. A inovação é considerada um elemento chave na economia baseada em conhecimento, e fundamental para a melhoria contínua da educação e para o aumento de resultados de aprendizagem [Looney 2009].

Em termos de inovações em processos (métodos, práticas e organização), as abordagens tradicionalistas de ensino são frequentemente caracterizadas como "transmissão direta", isto é, professores comunicam conhecimento de um modo estruturado, demonstram e explicam soluções. As abordagens de ensino-aprendizagem inovadoras, por outro lado, são caracterizadas como centradas no estudante, ou construtivistas. A ênfase está no desenvolvimento do pensamento e raciocínio (habilidades para "aprender-a-aprender") [OECD 2014]. Nestas, os professores assumem papéis diferentes, movendo-se do ensino "na frente da sala de aula" para 
participação ativa no processo de aprendizagem com os alunos, enquanto estes conduzem suas próprias investigações e desenvolvem soluções.

No entanto, segundo a OECD (2014), a inovação manifesta-se em diversas outras dimensões da educação, tais como em práticas instrucionais, estilos de ensino, organização do espaço educacional, objetos educacionais, métodos de avaliação, uso de computadores, abordagens de educação especial, colaboração entre professores, mecanismos de feedback, e gestão da escola como um todo.

\subsection{Aprendizagem Baseada em Competências e Outras Abordagens Educacionais Contemporâneas Compatíveis}

Dentre as abordagens educacionais que se apresentam como alternativas ao modelo tradicional, está a Aprendizagem Baseada em Competências, um paradigma que vem ganhando visibilidade desde a década de 90. Competência, neste sentido, é entendida como a combinação de conhecimentos (saber), habilidades (fazer) e atitudes (querer) necessárias à experiência efetiva da inovação [Mendonça 2007]. Este paradigma contrasta com a formação padronizada, na qual alunos adquirem uma base comum de conhecimentos que nem sempre o preparam para problemáticas reais [Mendonça 2007].

No contexto escolar, o conceito de competência passou a ser usado (1) como eixos de desempenho que alunos devem atingir no final de um determinado processo de aprendizagem, e (2) como forma de preparar os alunos para respostas eficazes na resolução de situações inéditas [Mendonça 2007]. Embora encontrem duras críticas no campo da educação [Hager et al. 1994], as abordagens que buscam articulação de saberes em prol da resolução de problemas em situações práticas retornaram ao foco, e passaram a ser evoluídas com mais ênfase nos últimos 10 anos. Exemplos destas abordagens são a Aprendizagem Vivencial de Kolb (1984) e a Aprendizagem Baseada em Problemas (PBL - do inglês Problem Based Learning) [Savery e Duffy 2001].

Segundo a teoria da Aprendizagem Vivencial de Kolb (1984), a aprendizagem (1) é um processo contínuo fundamentado na experiência individual; (2) requer uma resolução de conflitos entre modelos dialeticamente opostos de adaptação ao mundo; (3) deve ser concebida como um processo holístico de interação entre o indivíduo e o seu ambiente, que varia em extensão através do tempo e do espaço, e provê pontes conceituais entre elaborações teóricas e situações do dia-a-dia; e (4) é um processo de criação individualizada e personalizada de competências. De forma simplificada, Kolb (1984) prescreve um ciclo de criação de conhecimento que se inicia na experiência concreta dos indivíduos, gera questionamentos e reflexões a partir das observações da prática, passa por um processo de geração de conceitos abstratos e generalizações e encerra-se no teste dos conceitos e implicações inferidas sobre novas situações.

Já a Aprendizagem Baseada em Problemas (PBL) é uma abordagem instrucional centrada no estudante, que o ajuda a desenvolver o raciocínio e a comunicação, habilidades essenciais para sua vida profissional [Savery e Duffy 2001]. O PBL se desenvolve em torno de um problema para iniciar, direcionar e motivar o aprendizado de conceitos que levarão à sua solução. Ao contrário do ensino tradicional, no PBL a apresentação do problema antecede a exposição de conteúdo. O estudante é o protagonista da construção do seu aprendizado, que acontece então em um ambiente que o imerge em atividades sobre as quais recebe feedback constante de seus colegas. 
Ao passo que buscam a solução, os alunos ainda desenvolvem habilidades de trabalho em grupo, aprendizagem autônoma e atitudes tais como cooperação, ética e respeito pela opinião alheia. Além disso, adquirem a informação de forma integrada com os problemas vivenciados, contribuindo assim para que a aprendizagem seja mais significativa do ponto de vista da assimilação do conhecimento [Ausubel 1968]. Embora mais prática e operacional do que a aprendizagem vivencial, ambas são igualmente compatíveis com o paradigma da aprendizagem baseada em competências.

\subsection{Formação de Professores como Estratégia para Difusão de Inovações Educacionais}

A utilização de paradigmas de ensino-aprendizagem alternativos ao modelo tradicional como os discutidos na seção anterior, fomentando práticas inovadoras em educação, depende fortemente da iniciativa dos professores, apoiada por políticas públicas e pela gestão escolar. Porém, para que os professores adquiram, eles próprios, as competências necessárias para concretizar essa transformação, faz-se necessária formação adequada.

No Brasil, a formação dos professores da educação básica se dá nos cursos de Pedagogia e Licenciaturas, e vem sendo contínua e intensamente discutida ao longo dos anos, provocando a criação de diretrizes e políticas públicas específicas, como a Política Nacional de Formação de Profissionais do Magistério da Educação Básica (instituída através do Decreto 6.755 de 29 de janeiro de 2009 [Brasil 2009], revogado pelo Decreto 8.752 de 9 de maio de 2016 [Brasil 2016]), e as Diretrizes Curriculares Nacionais para a formação inicial em nível superior e formação continuada nos cursos de licenciatura e formação pedagógica [Brasil 2015].

Embora a política nacional de formação de professores [Brasil 2016] vise a melhoria da qualidade da educação, incluindo a atualização tecnológica dos profissionais, o termo inovação não aparece neste documento. As diretrizes curriculares para os cursos de licenciatura tocam vagamente nesta questão, afirmando que: "Compreende-se a docência (...) envolvendo conhecimentos específicos, interdisciplinares e pedagógicos, (...) que se desenvolvem na construção e apropriação dos valores éticos, linguísticos, estéticos e políticos do conhecimento inerentes à sólida formação científica e cultural do ensinar/aprender, à socialização e construção de conhecimentos e sua inovação (...)" [Brasil 2015, p. 3, Art. $2^{\circ} \S 1^{\circ}$, ênfase dos autores]; e visando "conduzir o(a) egresso(a): (...) às dinâmicas pedagógicas que contribuam para o exercício profissional (...), possibilitando as condições para o exercício do pensamento crítico, a resolução de problemas, o trabalho coletivo e interdisciplinar, a criatividade, a inovação, a liderança e a autonomia" [Brasil 2015, p. 6, Art. 50, ênfase dos autores]. De forma um pouco mais específica, o Programa Institucional de Bolsas de Iniciação à Docência (PIBID) [CAPES 2010] tem entre seus objetivos "inserir os licenciandos no cotidiano de escolas (...), proporcionando-lhes oportunidades de criação e participação em experiências metodológicas, tecnológicas e práticas docentes de caráter inovador e interdisciplinar que busquem a superação de problemas identificados no processo de ensino aprendizagem" (p. 26, ênfase dos autores).

Não fica claro, entretanto, como se espera que licenciados e pedagogos formem competências que lhes permitam inovar em educação, visto que não há diretrizes específicas que encorajem a inserção de disciplinas e vivências voltadas a essa questão 
nos currículos dos respectivos cursos superiores. O próprio uso de tecnologias, por exemplo, é quase ausente do currículo de formação dos futuros professores brasileiros, o que frequentemente cria uma resistência por parte dos licenciados em integrar inovações tecnológicas a sua prática docente [Lima e Loureiro, 2015].

Muito além de capacitar os educadores a utilizar recursos tecnológicos em sala de aula, um programa de formação de professores voltados à inovação deve ser capaz de garantir minimamente o desenvolvimento de conhecimentos sobre processos de inovação, habilidades de solução criativa de problemas e atitudes empreendedoras como parte do próprio processo. De acordo com Institute for the Future [IFTF 2008], as escolas tornar-se-ão no futuro sistemas dinâmicos em rede, capazes de renovar-se com as mudanças. Mas, para isso, os educadores precisam desenvolver as competências necessárias para catalisar estas mudanças de forma colaborativa.

\section{Panorama de Programas de Pós-Graduação em Educação e Tecnologia}

Para conduzir esta análise, selecionamos programas de pós-graduação em educação e tecnologia no Brasil e nas principais universidades do mundo. Com o auxílio da ferramenta Keyword Density Analyzer1, analisamos os termos mais frequentes (e suas respectivas densidades) utilizados na descrição dos objetivos dos programas e nas suas respectivas disciplinas. Os 12 programas nacionais selecionados foram encontrados através de buscas sistemáticas no site da CAPES, e arbitrárias na internet, conduzidas em Setembro/2015. Os programas internacionais foram consultados nos sites das próprias instituições, seguindo o ranking do tema "Educação" no site TOP Universities (2015). Portanto é provável que esta não seja uma lista exaustiva, mas é suficientemente representativa para traçar um panorama. Para conduzir esta análise, selecionamos 8 (oito) programas de pós-graduação em educação, de 7 (sete) universidades do ranking citado. A Tabela 1 lista todos os programas analisados.

Tabela 1 - Programas de pós-graduação em Educação e Tecnologia

\begin{tabular}{|c|c|c|c|}
\hline \multirow{6}{*}{$\begin{array}{l}\text { Programas } \\
\text { Nacionais }\end{array}$} & Inovação em Tecnologias & \multicolumn{2}{|l|}{ Tecnologias Interativas } \\
\hline & Educacionais (Anhembi & \multirow{2}{*}{$\begin{array}{l}\text { Aplicadas à Educação (PUC- } \\
\text { SP) }\end{array}$} & Tecnologia e Gestão em \\
\hline & Novas tecnologias para & & \\
\hline & $\begin{array}{l}\text { aprendizagem no ensino médio } \\
\text { e fundamental (Gama Filho) }\end{array}$ & $\begin{array}{l}\text { Tecnologias na Aprendizagem } \\
\text { (SENAC) }\end{array}$ & $\begin{array}{l}\text { Tecnologias Educacionais em } \\
\text { Rede (UFSM) }\end{array}$ \\
\hline & $\begin{array}{l}\text { Tecnologias Educacionais e } \\
\text { Educação a Distância (IFRN) }\end{array}$ & $\begin{array}{l}\text { Educação Inovadora: didática, } \\
\text { design e autoria } \\
\text { (Singularidades) }\end{array}$ & $\begin{array}{l}\text { Tecnologias, Comunicação e } \\
\text { Educação (UFU) }\end{array}$ \\
\hline & $\begin{array}{l}\text { Informática na Educação } \\
\text { (IFRS) }\end{array}$ & $\begin{array}{l}\text { Informática na Educação } \\
\text { (UFRGS) }\end{array}$ & $\begin{array}{l}\text { Educação e Novas Tecnologias } \\
\text { (UNINTER) }\end{array}$ \\
\hline \multirow{6}{*}{$\begin{array}{l}\text { Programas } \\
\text { Internacionais }\end{array}$} & University-led Teacher & & \\
\hline & Training (University College & Technology, Innovation, and & The Stanford Teacher education \\
\hline & $\begin{array}{l}\text { London) } \\
\text { Learning Design and }\end{array}$ & $\begin{array}{l}\text { Education (Harvard University) } \\
\text { Master of Education and }\end{array}$ & program (Stantord Unıversity) \\
\hline & Technology & Graduate Certificate in & \\
\hline & (Stanford University) & $\begin{array}{l}\text { Education (The University of } \\
\text { Melbourne) }\end{array}$ & $\begin{array}{l}\text { Master of Education (The } \\
\text { University of Hong Kong) }\end{array}$ \\
\hline & $\begin{array}{l}\text { MSc Education - Learning and } \\
\text { Technology (University of } \\
\text { Oxford) }\end{array}$ & \multicolumn{2}{|c|}{$\begin{array}{l}\text { UCLA Teacher Education Program (University of California, Los } \\
\text { Angeles) }\end{array}$} \\
\hline
\end{tabular}

\footnotetext{
${ }^{1}$ http://tools.seobook.com/general/keyword-density/
} 
Dentre os programas nacionais, 04 ocorrem na modalidade a distância, 06 presencialmente e 02 não informam a modalidade. Para 07 deles, não encontramos a informação sobre a carga horária, enquanto os outros variavam entre 360 horas (04 cursos) e 440 horas (01 curso). A quantidade de disciplinas também apresentou uma grande variação, entre 10 (Instituto Singularidades) e 27 disciplinas (UFRGS). A Tabela 2 mostra os termos de densidade maior que 5 identificados nos objetivos (total de 71 termos) e conteúdo (total de 68 termos). Percebe-se que os seus objetivos e disciplinas citam tecnologia e educação na mesma proporção. As disciplinas dão uma clara ênfase à educação, e a intervenções tecnológicas educacionais bem consolidadas, especialmente a educação a distância.

Tabela 2 - Análise da Densidade de palavras-chave

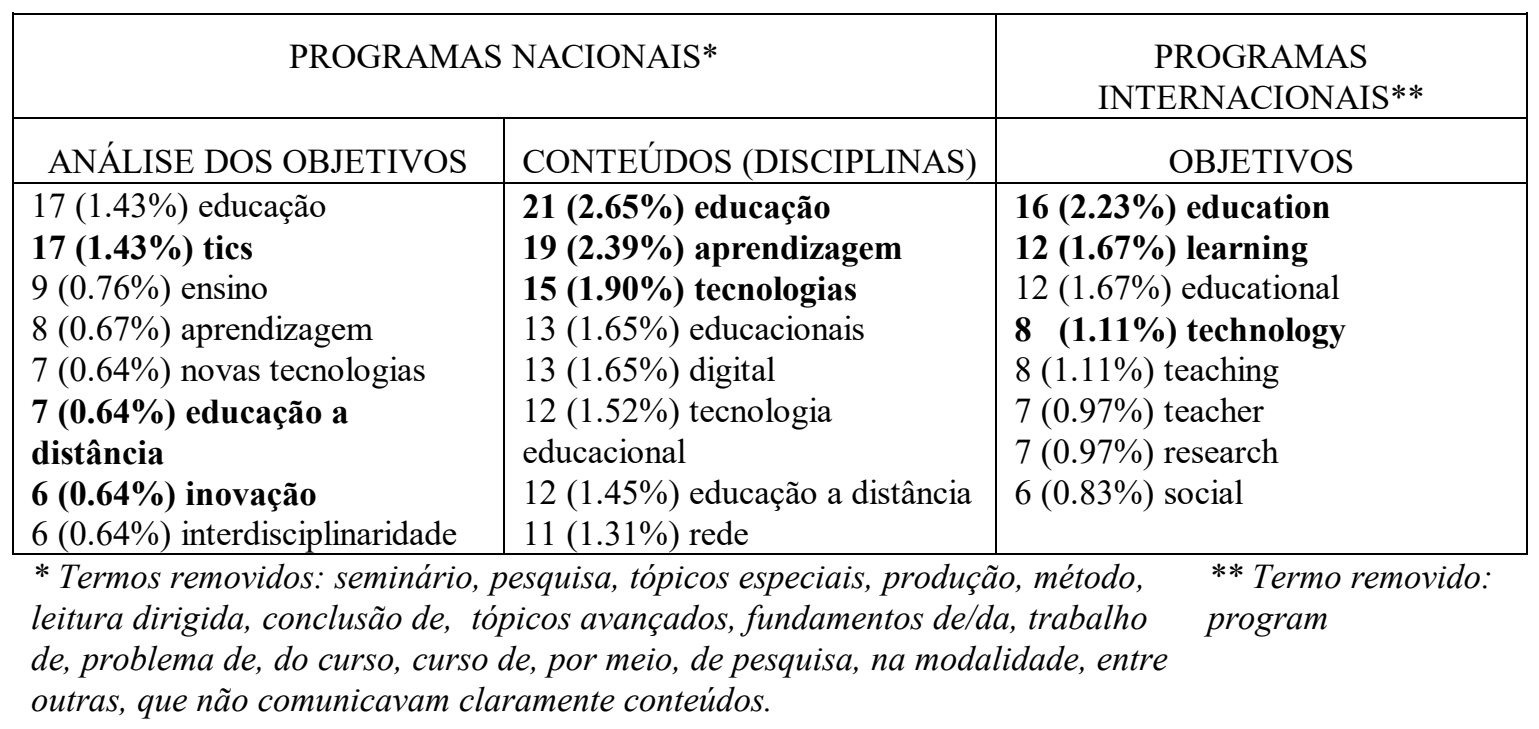

Os programas internacionais, por sua vez, seguem a modalidade presencial. Quase todas as universidades possuem programas alternativos de carga horária menor, embora tenhamos focado nos mestrados. Nem todos os cursos apresentam em suas páginas a carga horária ou as disciplinas de seu programa, por isso analisamos apenas os seus objetivos. A Tabela 1 apresenta os termos de densidade maior que 5 identificados nos objetivos dos programas (total de 441 termos).

Percebe-se que os objetivos citam alguns dos termos vistos dentre as instituições brasileiras que são chave para o curso proposto, tais como educação, aprendizagem e tecnologia. Porém, a partir dos objetivos observa-se que há um foco maior em educação (2,23\% para education e $1,67 \%$ para educational) .

Dentre os termos encontrados, inovação aparece citado nos objetivos de 04 programas nacionais, no entanto só aparece como disciplina em dois destes cursos. No curso do Instituto Singularidades, em particular, conteúdos relacionados à inovação são encontrados em três disciplinas diferentes. De fato, este foi o único programa nacional, dentre os analisados, que aborda explicitamente conteúdos relacionados a processos de inovação. Pesquisas exploratórias dentre esses programas permitiram identificar que a Universidade de Harvard, em seu programa Technology, Innovation, and Education, apresenta inovação como um ponto de destaque para área de educação, sendo o único programa estrangeiro, dentre os analisados, que aborda explicitamente conteúdos relacionados a processos de inovação. 
V Congresso Brasileiro de Informática na Educação (CBIE 2016)

Anais do XXII Workshop de Informática na Escola (WIE 2016)

\section{Proposta Curricular do EITA}

\subsection{Sobre a Estratégia Pedagógica do Curso}

A proposta do curso de Educação, Inovação e Tecnologia Aplicada (EITA) nasce da necessidade de aprofundar e conectar competências relacionadas à inovação, por meio do uso de recursos de tecnologia da informação e comunicação (TIC), visando expandir a capacidade de educadores utilizarem metodologias eficientes para empreenderem inovações. Os problemas a serem trabalhados no curso serão desafios do contexto profissional dos próprios alunos, que vivenciarão cargas horárias teórica e prática de Educação, Inovação e Tecnologia, e buscarão selecionar e aplicar as técnicas mais adequadas para gerar uma solução inovadora para o problema proposto, no formato de uma sequência didática.

A metodologia deste curso está fundamentada em bases filosóficas e epistemológicas advindas do construtivismo, em particular da teoria da aprendizagem vivencial [Kolb 1984], a ser implementada por meio do método instrucional de aprendizagem baseada em problemas (PBL) e do processo de Design Thinking [Brown 2008] (Figura 1).

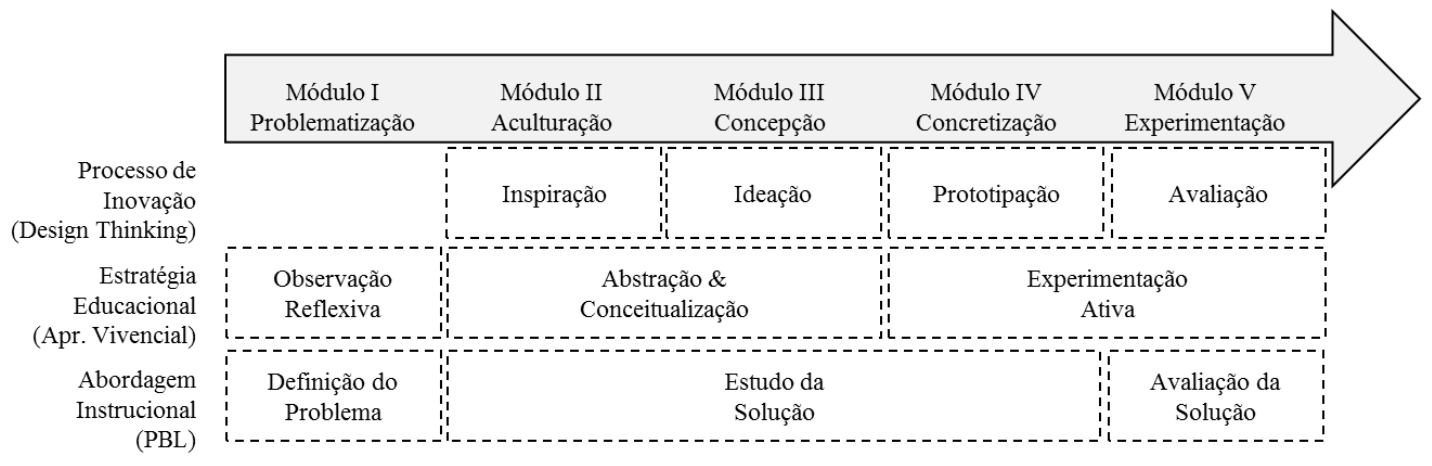

Figura 1 - Concepção geral do EITA

O Design Thinking vem recentemente sendo adaptado e aplicado com sucesso na educação, como um processo criativo que busca soluções significativas em torno especificamente da comunidade escolar [IDEO s.d.]. O processo é organizado nas etapas de: (1) inspiração - imersão na realidade dos sujeitos e identificação de problemas; (2) ideação - etapa criativa incluindo avaliação de alternativas e geração de soluções inovadoras apropriadas; e (3) implementação - experimentação prática e avaliação para adequação e melhoria contínua da solução. Neste contexto, enxergamos uma oportunidade para integração das três metodologias citadas, para condução de um curso com foco no desenvolvimento de competências para a geração de soluções inovadoras no contexto da educação.

\subsection{Sobre o Conteúdo Programático}

A interdisciplinaridade é um dos pilares de constituição do currículo deste curso, uma vez que ele próprio é classificado como multidisciplinar, envolvendo temas da educação e das TICs. O conteúdo programático abrange disciplinas que se costuram entre os módulos do curso para assegurar que os alunos exercitarão habilidades necessárias para condução de atividades das etapas específicas do processo de criação 
V Congresso Brasileiro de Informática na Educação (CBIE 2016)

Anais do XXII Workshop de Informática na Escola (WIE 2016)

(Tabela 3). Em particular, a disciplina de Práticas de Criação tem o objetivo de proporcionar aos alunos oportunidades de exercitar em sala de aula os conceitos abordados, apresentar a evolução dos projetos, discutir e construir colaborativamente.

Assim, como meio para superar a fragmentação do conhecimento e conectar de forma consistente as disciplinas de diferentes áreas, os alunos desenvolverão um projeto único, seguindo a abordagem PBL. Este projeto contará para avaliação em todas as disciplinas, visto que seus diversos módulos corresponderão a diferentes disciplinas, demandando evidências de construção de conhecimentos e habilidades de cada uma delas. Espera-se que o desenvolvimento deste projeto possibilite a integração sistêmica dos conhecimentos adquiridos nas diferentes disciplinas.

Tabela 3 - Disciplinas do EITA

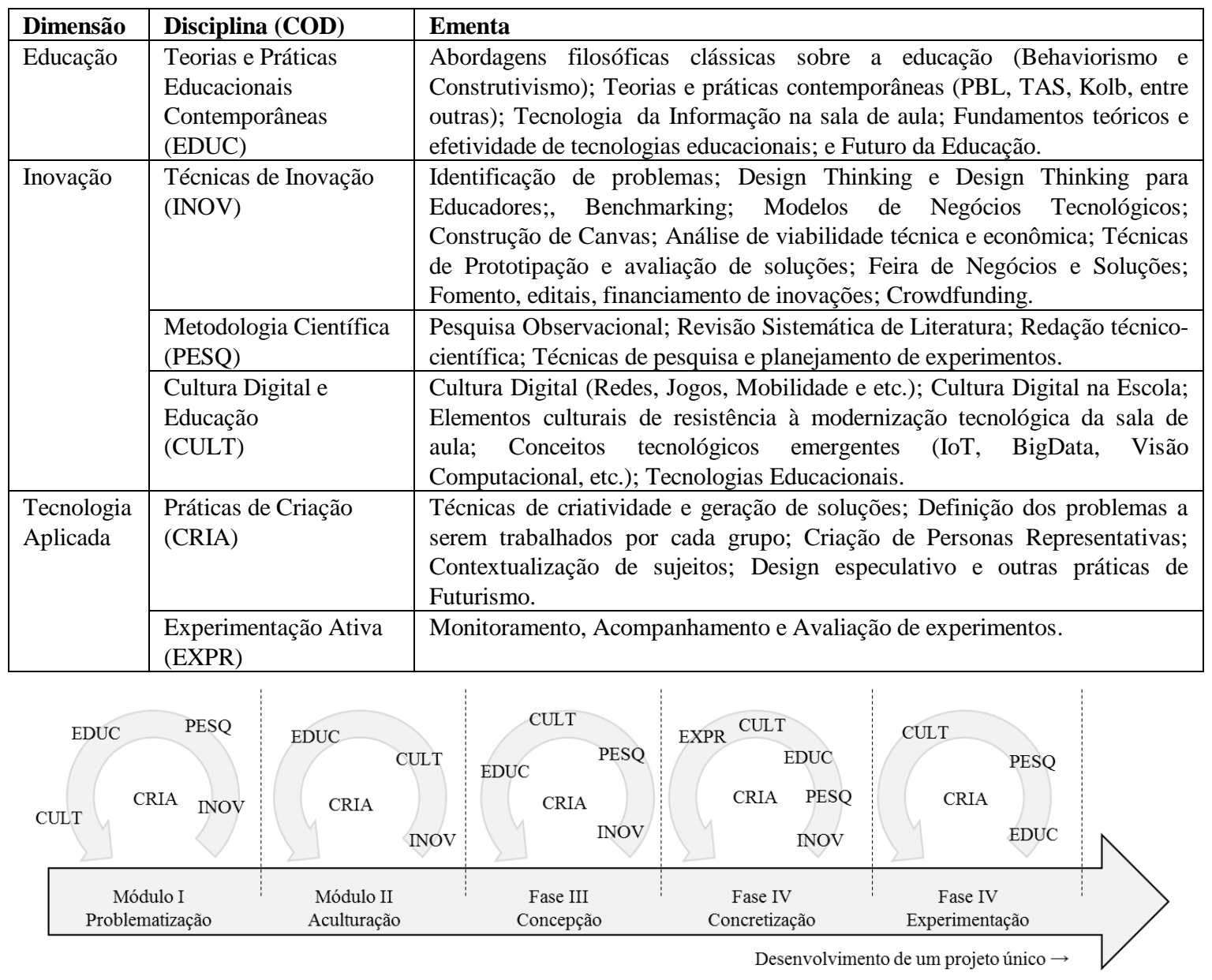

Figura 2 - Encadeamento das disciplinas (vide códigos na Tabela 3)

A carga horária das disciplinas distribuída pelos diferentes módulos também proporciona uma articulação proativa e planejada entre professores, para que os seus conteúdos interajam de forma cíclica e incremental. A Figura 2 ilustra o encadeamento entre as disciplinas ao longo do curso e do desenvolvimento do projeto. O curso contará também com diversas interações extra-classe, dentre elas: (a) debate aberto ao público sobre o futuro da educação, conduzido pelos alunos; (b) execução de pesquisa de campo baseada num plano de observação, para identificação de problemas e aprofundamento do conhecimento sobre os perfis de alunos que serão alvo das 
V Congresso Brasileiro de Informática na Educação (CBIE 2016)

Anais do XXII Workshop de Informática na Escola (WIE 2016)

inovações a serem desenvolvidas; (c) organização e execução de uma Feira de Negócios, para agregar e dar publicidade a produtos e serviços relacionados aos projetos propostos pelos alunos; e (d) experimentação prática da sequências didáticas propostas.

\section{Conclusão}

A inovação do sistema educacional depende, em grande parte, da iniciativa dos professores. No entanto, para sentirem-se empoderados para inovar, os professores necessitam de uma formação que contemple competências adequadas o que, de acordo com os dados que apresentamos neste artigo, ainda não se vê nos cursos de Pedagogia e licenciatura no Brasil. Na tentativa de colaborar para o empoderamento dos professores da educação básica, e assim promover, através deles, mudanças de paradigma e estrutura nas escolas, propomos um curso de pós-graduação em Educação, Inovação e Tecnologia Aplicada (EITA). O curso é, ele próprio, baseado em uma abordagem inovadora, com disciplinas que dialogam de forma entrelaçada em torno de um projeto único, promovendo a aprendizagem vivencial através de uma metodologia baseada em problemas reais trazidos pelos próprios aprendizes. Para a resolução destes problemas, propõe-se o método do Design Thinking, com o objetivo final de gerar uma solução viável no formato de uma sequência didática inovadora, que possa ser divulgada e compartilhada nas comunidades escolares, estimulando assim o surgimento de iniciativas similares.

O curso proposto encontra-se em fase de iniciação e, portanto, ainda não existem registros dos seus resultados práticos. Em trabalhos futuros, pretendemos acompanhar, avaliar, e relatar em detalhes o impacto causado nos participantes do curso e pelos participantes do curso em seus locais de trabalho (escolas, universidades, etc.).

\section{Referências}

Ausubel, D. P.; Novak, J.D.; Hanesian, H. (1978) "Educational psychology: A cognitive view". Holt, Rinehart and Winston.

Brasil, Presidência da República (2009) Decreto 6.755 de 29 de janeiro de 2009. Disponível em: http://www.planalto.gov.br/ccivil_03/_ato20072010/2009/decreto/d6755.htm. Último acesso: 29 de maio de 2016.

Brasil, Ministério da Educação, Conselho Nacional de Educação (2015) Resolução n.2 de lo de julho de 2015.

Brasil, Presidência da República (2016) Decreto 8.752 de 9 de maio de 2016. Disponível em: http://www.planalto.gov.br/ccivil_03/_Ato20152018/2016/Decreto/D8752.htm\#art19. Último acesso: 29 de maio de 2016.

Brown, T. (2008) Design Thinking. Harvard Business Review. June.

CAPES, Coordenação de Aperfeiçoamento de Pessoal de Nível Superior (2010) Portaria n. 72, de 9 de abril de 2010, Diário Oficial da União, Seção 1, n. 68, 12 de abril de 2010 .

Hager, P., Gonczi, A. Athanasou, J. (1994) General Issues about Assessment of Competence, Assessment \& Evaluation in Higher Education, 19: 1, 3 - 16. 
V Congresso Brasileiro de Informática na Educação (CBIE 2016)

Anais do XXII Workshop de Informática na Escola (WIE 2016)

IDEO (s.d.) Design Thinking for Educators. Riverdale-IDEO. Disponível em $<$ http://www.designthinkingforeducators.com/>. Último acesso em 25-01-2015.

IFTF - Institute For The Future (2008) 2020 Forecast: Creating the Future of Learning. Disponível em: http://www.iftf.org/iftf-you/programs-initiatives/future-of-learning/. Último acesso: 29-05-2016.

Kelley, D.; Singer, S.; Herrington, M.; GEM Global Report 2015/16. Disponível em: http://www.gemconsortium.org/report/49480. Último acesso: 29-05-2016.

Kenski, V. M. (2015) A urgência de propostas inovadoras para a formação de professores para todos os níveis de ensino. Rev. Diálogo Educ., v.15, n.45, p. 423441, maio/ago.

Kolb, D. A. (1984) "Experiential learning: experience as the source of learning and development". Englewood Cliffs, NJ: Prentice Hall.

Leal, A. D. C.; Neponucemo, C. C. ; Oliveira, J. G. ; Lira, W. S. ; Ramalho T. C. S. (2011) Diagnóstico da Tendência Empreendedora do Corpo Docente de uma Instituição Pública de Ensino Superior, Anais do VIII Simpósio de Excelência em Gestão e Tecnologia, Rio de Janeiro.

Lima, L. Loureiro, R. (2015) A integração entre Docência e Tecnologias Digitais da Informação e Comunicação na Formação de Licenciandos. Anais do XXI Workshop de Informática na Escola - WIE, Maceió - AL.

Looney, J. W. (2009) “Assessment and Innovation in Education", OECD Education Working Papers, No. 24, OECD Publishing. DOI: 10.1787/222814543073

Mazzioni, Sad, “As Estratégias Utilizadas No Processo De Ensino-Aprendizagem: Concepções De Alunos E Professores De Ciências Contábeis" Revista Eletrônica de Administração e Turismo - ReAT | vol. 2 - n. 1 - JAN./JUN. - 2013.

Mendonça, M. E. N. (2007) Aprendizagem e Avaliação de Competências na Escola Moderna. Dissertação apresentada ao Mestrado em Ciências da Educação, Área da Inovação Pedagógica, Universidade da Madeira.

Núcleo de Informação e Coordenação do Ponto BR - NIC.br (2016). Educação e tecnologias no brasil : um estudo de caso longitudinal sobre o uso das tecnologias de informação e comunicação em 12 escolas públicas. São paulo : Comitê Gestor da Internet no Brasil.

OECD (2005) "Oslo Manual - Guidelines for Collecting and Interpreting Innovation Data”. OECD Publishing. ISBN 92-64-01308-3.

OECD (2014) "Measuring Innovation in Education: A New Perspective, Educational Research and Innovation", OECD Publishing. DOI: 10.1787/9789264215696-en.

Savery, J. R., Duffy, T. M. (2001) Problem Based Learning: An instructional model and its constructivist framework. CRLT Technical Report No. 16-01. Indiana University.

TOP Universities (2015). QS World University Rankings by Subject 2015 - Education. Disponível em < www.topuniversities.com > Último acesso: 30-05-2016. 